Zhu Liu, Fengming Xi Institute of Applied Ecology, Chinese Academy of Sciences, Shenyang, China.

liuzhu@iae.ac.cn

Dabo Guan University of Leeds, Leeds, UK.

\section{Sexism: measure journal objectivity}

Your initiative to address the issue of sexism in publishing is laudable (Nature 491, 495; 2012). But scientific editors may be biased in other ways that influence the publication process - and they are not in the best position to recognize and correct their own biases.

An independent, external assessment body that regularly evaluates editorial practices across scientific journals might be the answer. This would promote transparency and reassure authors that their work is being dealt with fairly. An 'objectivity factor' resulting from such an assessment could become a key metric of journal performance, alongside its impact factor.

Boyan K. Garvalov University of Giessen, Germany.

boyan.garvalov@patho.med.unigiessen.de

\section{Sexism: control experimental bias}

Implicit bias against women (Nature 491, 495; 2012) has a record of influencing the design of experiments and collection of data in the life and mind sciences.

Over the past four decades, feminist scientists, historians and philosophers of science have presented case study after case study showing how sexist bias can distort scientific results. Examples include work by Rebecca JordanYoung on brain-organization theory, Anne Fausto-Sterling on the biological study of sex differences and Anelis Kaiser on neuroimaging.

In the interest of greater accuracy, controls for sexist bias need to be more rigorous. Letitia Meynell Dalhousie University, Halifax,
Nova Scotia, Canada.

letitia.meynell@dal.ca

\section{Sexism: conferences should seek a balance}

Conference committees and symposia chairs should follow Nature's lead and be more open and proactive about gender balance (Nature 491, 495; 2012).

Publishing an online declaration of gender-balance policy would help. Data from each of the past, say, three meetings could also be posted online to indicate the percentage of women who were registrants, invited plenary and keynote speakers, session speakers, programme committee members, or session chairs. If there is a significant gap between the percentage of women attending the conference and the overall percentage of women speakers or committee members, then a policy overhaul may be called for

Jennifer L. Martin University of Queensland, Brisbane, Australia. j.martin@imb.uq.edu.au

\section{More trials needed to assess sleeping pills}

Sam Fleishman suggests that the controlled use of sleeping pills helps to counter the lifedisrupting consequences of insomnia (Nature 491, 527; 2012). But, to our knowledge, there is no convincing evidence that sleep medication can reactivate the health-restoring functions of sleep.

Good-quality sleep improves cognitive performance, vigilance, memory and mood. Poor sleep is associated with potentially damaging physiological effects such as inflammation (M. R. Irwin et al. Brain Behav. Immun. 24, 54-57; 2010), compromised immune-cell activity (E. Fondell et al. Brain Behav. Immun. 25, 1367-1375; 2011) and telomere shortening (A. A. Prather et al. J. Aging Res. 2011, 721390; 2011).

However, the regular use of sleeping pills has been linked with increased mortality and morbidity from infection, depression or cancer (see, for example, D. F. Kripke et al. BMJ
Open 2, e000850; 2012).

Large randomizing trials are needed to evaluate the benefits and risks of sleeping pills so that they can be prescribed more responsibly.

Maximilian Moser Medical University of Graz, Austria max.moser@medunigraz.at Daniel F. Kripke University of California San Diego, California, USA.

\section{Toe-clipping vital to amphibian research}

Keeping a record of the global decline of amphibian populations depends on the identification and marking of individuals in the field, commonly by toe-clipping. This work is under threat: the Brazilian federal government and non-governmental organizations want to prohibit toe-clipping without scientific justification.

Toe-clipping is a simple, cost-effective, minimally invasive marking technique that has been in use for decades in herpetological research. The Brazilian federal agency that enforces environmental policies, IBAMA, is claiming that the practice is a form of mutilation and should be a criminal offence under federal law (see go.nature. com/qkij7l; in Portuguese). The Brazilian Herpetological Society has protested against this anthropocentric position (see go.nature.com/thpwdg; in Portuguese) on the grounds that it would set back efforts to understand amphibian decline.

Brazil is home to the greatest amphibian diversity on Earth, but knowledge of population dynamics is scant and will remain so without access to reliable marking methods. We acknowledge that toe-clipping is not an ideal solution but, when properly implemented, it has minimal effects on amphibian survival and behaviour. Without this technique, it will be harder to obtain crucial information that could prevent amphibian species from becoming extinct (W. C.

Funk et al. Nature 433, 193; 2005).

Décio T. Corrêa ${ }^{\star}$ State

University of Campinas, São

Paulo, Brazil.

decio.tadeu@gmail.com
${ }^{\star}$ On behalf of 5 co-signatories (see go.nature.com $/ 5 \mathrm{sgsqo}$ for full list).

\section{Missing data mean holes in tree of life}

As part of the Open Tree of Life project (http://opentreeoflife. org), we surveyed publications covering all domains of life and found that most phylogenetic trees and nucleotide alignments from the past two decades have been irrevocably lost.

Of 6,193 papers we surveyed in more than 100 peer-reviewed journals, only $17 \%$ present accessible trees and alignments (used to infer relatedness). Contacting lead authors to procure data sets was only $19 \%$ successful. DNA sequences were deposited in GenBank for almost all these studies, but it is the actual character alignments that are pivotal for reproducing phylogenetic analyses. We estimate that more than $64 \%$ of existing alignments or trees are permanently lost.

This problem will increasingly hinder phylogenetic inference as the use of whole-genome data sets becomes common. Journals need to reinforce a policy of online data deposition, either as supplementary material or in repositories such as TreeBASE

(http://treebase.org) or

Dryad (http://datadryad.org) including for data sets based on previously published sequences. Ecologists, evolutionary biologists and others will then have access to rigorous phylogenetics for testing their hypotheses.

Bryan T. Drew ${ }^{\star}$ University of Florida, Gainesville, USA.

bdrew@ufl.edu

${ }^{*}$ On behalf of 8 co-signatories (see go.nature.com/gzqcr9 for full list).

\section{CORRECTION}

The Outlook article 'Genetics: Searching for answers' (Nature 491 (suppl. 7422), S4-S6; 2012) incorrectly stated that the Simons Simplex Collection consists of 3,000 blood samples taken from more than 700 people. In fact, it contains 13,000 blood samples taken from 3,000 people. 